\section{STROMAL REMODELING REGULATES DENDRITIC CELL ABUNDANCE IN THE TUMOR MICROENVIRONMENT}

${ }^{1}$ Athanasios Papadas*, ${ }^{1}$ Gauri Deb, ${ }^{1}$ Adam Officer, ${ }^{2}$ Chelsea Hope, ${ }^{2}$ Philip Emmerich, ${ }^{1}$ Alexander Cicala, ${ }^{1} J o s h u a$ Wiesner, ${ }^{1}$ Garrett Arauz, ${ }^{2}$ Adam Pagenkopf, ${ }^{2}$ Kristina Matkowskyj, ${ }^{2}$ Dustin Deming, ${ }^{3}$ Katerina Politi, ${ }^{4}$ Scott Abrams, ${ }^{1}$ Olivier Harismendy, ${ }^{1}$ Fotis Asimakopoulos. ${ }^{1}$ UCSD, La Jolla, CA, USA; ${ }^{2}$ UW-Madison, Madison, WI, USA; ${ }^{3}$ Yale, New Haven, CT, USA; ${ }^{4}$ Roswell Park, Buffalo, NY, USA

Background Stimulatory dendritic cells (SDC), enriched within the Batf3-DC lineage (also known as conventional type 1 DC, cDC1), engage in productive interactions with $\mathrm{CD} 8+$ effectors along tumor-stroma boundaries. This puzzling pattern of Tcell-DC localization has been interpreted as "tumor-exclusion", limiting anti-tumor immunity. To understand this paradox, we hypothesized that dynamic matrix remodeling at the invasive margin generates unique activation and cell-fate cues critical for Batf3-DC homeostasis.

Methods We studied immunocompetent tumor models of lung carcinoma, breast carcinoma, melanoma and multiple myeloma. For mechanistic experiments, we generated novel Vcantargeted models through CRISPR-Cas9 targeting. We delineated DC subsets through multi-parametric flow cytometry and tumor immune contexture through mass cytometry. Batf3-DC cellular models included MutuDC1940 immortalized DC and iCD103 primary cells. TCGA data were mined for human validation.

Results We find that CD8+ T cells massively infiltrate tumor matrices undergoing robust matrix proteoglycan versican (VCAN) proteolysis, an essential organ-sculpting modification in development and adult tissue-plane forging. Across 7591 samples from 20 TCGA cancer types, a significant-positive correlation between VCAN substrate expression and Batf3-DC score was observed, suggesting that the VCAN pathway may regulate Batf3-DC across several cancer types. Experimental Vcan depletion in the tumor microenvironment was detrimental for Batf3-DC. Batf3-DC abundance was restored through the VCAN N-terminal fragment (matrikine) versikine, physiologically generated through ADAMTS protease activity in remodeled stroma. In addition to Batf3-DC expansion, versikine resulted in G-MDSC contraction as well as the emergence of an atypical innate lymphoid (NK/ILC1) subset expressing cytotoxicity receptors, low IFNgamma and robust pro-survival GM-CSF. Despite broad intratumoral IRF8 induction (10-100-fold), adoptive transfer of pre-DC into versikinereplete microenvironments did not influence their differentiation choice between Batf3-DC and CDC2. Instead, versikine delivered a distinct Batf3-DC activation signal characterized by non-TLR maturation as well as downregulation of TGFbeta and Wnt signaling. In vivo, versikine promoted Batf3-DC abundance through NK cells but independently of stromal TLR2 or CD44. Versikine sensitized immune-evasive tumors to STING agonist immunotherapy in a Batf3-DC dependent manner and promoted antigen-specific CD8 + responses. VersikineDC signatures correlated with $\mathrm{CD} 8+\mathrm{T}$ cell scores in human lung cancers.

Conclusions We demonstrate that dynamic extracellular matrix remodeling controls Batf3-DC abundance in the tumor microenvironment. N-terminal proteolysis of the matrix proteoglycan versican (VCAN), releases a bioactive fragment (matrikine), versikine, that is remarkably necessary and sufficient for Batf3-DC accumulation. Versikine orchestrates a multi-lineage network that regulates Batf3-DC activation and survival at matrix-remodeling interfaces. Therapeutic harnessing of matrix-Batf3-DC cross-talk sensitizes immuneevasive tumors to immunotherapy.

Acknowledgements We acknowledge support by the National Cancer Institute (R01CA252937 and U01CA196406), the American Cancer Society (127508-RSG-15-045-01-LIB), the Leukemia and Lymphoma Society (6551-18), the UW Trillium Myeloma Fund and the Robert J. Shillman Foundation.

Ethics Approval Laboratory animal work was performed under IACUC-approved protocols \#M5476 and \#S19109 in the University of Wisconsin-Madison and University of California, San Diego respectively.

http://dx.doi.org/10.1136/jitc-2021-SITC2021.936 